\title{
One-Dimensional Modeling of Transport in Small Stellarators
}

\author{
K. J. S. MERTENS, W. N. G. HITCHON, D. T. ANDERSON, AND J. L. SHOHET, fellow, IEeE
}

\begin{abstract}
An existing stellarator reactor transport code has been modified in order to model small stellarator experiments. Changes have been made in the treatment of the neutrals, the atomic physics processes, and the transport models. We have investigated the extent to which the hollow density profiles experimentally detected during electron cyclotron resonance heating (ECRH) experiments in the interchangeable module stellarator (IMS), a small modular stellarator, can be modeled. In this, we were guided by the experimental observation of large variations in potential around the magnetic surfaces, which can give rise to radially directed $E \times B$ convective flow. A diffusion model based on small-scale convective cell structures cannot simulate the experimental profiles. Including a purely convective term in the particle balance equation allows for a much more accurate modeling of the profiles. A comparison between numerical and experimental results is presented.
\end{abstract}

\section{INTRODUCTION}

$I^{1}$ N RECENT YEARS, a general trend in the numerical treatment of fluid transport in fusion devices has been towards "reactor" codes, with the incorporation of features such as neutral beam heating, pellet injection, and inclusion of several ionic species [1]. In our laboratory, however, experiments are carried out on relatively small stellarators, and the features mentioned above are quite irrelevant. Moreover, the degree of ionization $\eta$ of our plasmas is certainly not as high as in large-size devices, and the transport mechanisms can be considerably different from those in fully ionized reactor-sized plasmas. Finally, the sophistication present in a typical "reactor" transport code (for example, BALDUR [2]) is often not necessary for our applications.

For this reason, we present in this paper an exploration of the opposite case. WHISTEL, an existing one-dimensional stellarator reactor transport code [3], has been modified to simulate neutral-dominated low-temperature stellarator plasmas in a more realistic fashion.

The goal of this work is to investigate the nature of the measured hollow density profiles in the interchangeable module stellarator (IMS) [4]. These hollow profiles, which peak between the electron cyclotron and the upper hybrid

Manuscript received March 4, 1987; revised December 14, 1987. This research was supported in part by the Department of Energy under Grant DE-FG02-86ER53216.A001, by the National Science Foundation under Grant ECS-8514598, by Oak Ridge National Laboratory under Contract $19 X-27434 C$, and by the U.S. Department of Energy under Grant DEFG02-86ER53201.

The authors are with the Torsatron/Stellarator Laboratory, University of Wisconsin-Madison, Madison, WI 53706.

IEEE Log Number 8719473. resonance layers, have been observed over a wide range of experimental conditions.

To model these profiles accurately, the physics contained in the original code had to be adapted to the cooler and less dense plasmas prevalent in a small stellarator such as IMS. These changes will be discussed in the subsequent sections. In particular, the neutrals are treated zero dimensionally, in that their temperature, although time dependent, is independent of position. Atomic and molecular collision processes that are relevant at fusion temperatures are not necessarily so at low temperatures. Thus, some processes could be neglected, while others have been included.

Several different purely "diffusive"' transport models have been investigated, but none of them was satisfactory in explaining the profiles. By including particle convection as a new mechanism affecting the particle balance, much better agreement between experiment and simulation could be achieved. Numerical values for the convective velocity used in the code are based on experimental measurements.

Our main conclusion is that a model in which diffusive transport is combined with outward convection produces numerical results consistent with the experimentally observed profiles.

This convective flow is thought to be caused by potential variations around the magnetic flux surfaces which lead to poloidally directed electric fields and, combined with the toroidal magnetic field, to an $\boldsymbol{E} \times \boldsymbol{B}$ particle drift that is directed radially outward. These matters will only be touched upon briefly in this paper, and have been discussed more fully elsewhere [5].

Section II is a brief discussion of the transport equations that are solved numerically. The relevant physics included in the computer code is discussed in Section III. A comparison between experimental and numerical results is presented in Section IV, while conclusions can be found in Section V.

\section{Transport EQuations}

Our interest is focused upon the determination of ion and electron temperatures and densities. A set of equations governing these variables can be derived by taking velocity moments of the Boltzmann equation [6]. After averaging these equations over a magnetic flux surface, a set of fluid equations with a one-dimensional spatial vari- 
ation is obtained [7], [8]. The independent variable is a radial flux coordinate $r$, measured from the minor axis outwards. Thus, as pointed out in [3], the noncircular flux surfaces of stellarators are modeled as "equivalent circles."

In this fashion, the following set of partial differential equations is obtained [3] (for the case of one ion species): ${ }^{1}$

$$
\begin{gathered}
\frac{\partial n_{i}(r, t)}{\partial t}=-\frac{1}{r} \frac{\partial}{\partial r}\left(r \Gamma_{i \perp}\right)+S_{i}(r, t) \\
\frac{\partial}{\partial t}\left(\frac{3}{2} n_{e}(r, t) T_{e}(r, t)\right) \\
=-\frac{1}{r} \frac{\partial}{\partial r}\left[r\left(q_{e \perp}+\frac{3}{2} T_{e} \Gamma_{e \perp}\right)\right] \\
-Q_{e i}+P_{e, \mathrm{RF}}+P_{e, N} \\
\frac{\partial}{\partial t}\left(\frac{3}{2} n_{i}(r, t) T_{i}(r, t)\right) \\
=-\frac{1}{r} \frac{\partial}{\partial r}\left[r\left(q_{i \perp}+\frac{3}{2} T_{i} \Gamma_{i \perp}\right)\right]+Q_{e i}+P_{i, N}
\end{gathered}
$$

where

$$
\begin{aligned}
& r \text { radial coordinate, } \\
& t \quad \text { time, } \\
& n_{e}, n_{i} \quad \text { electron and ion densities }\left(\left[n_{e}\right]=\left[n_{i}\right]=\right. \\
& \mathrm{cm}^{-3} \text { ), } \\
& T_{e}, T_{i} \quad \text { electron and ion temperatures }\left(\left[T_{e}\right]=\left[T_{i}\right]\right. \\
& =\mathrm{eV} \text { ), } \\
& \Gamma_{e \perp}, \Gamma_{i \perp} \text { electron and ion particle fluxes perpendic- }
\end{aligned}
$$

\footnotetext{
'It is worthwhile to point out that to arrive at the flux-surface-averaged energy balance equations, (2) and (3), which are fairly standard (see, for example, [1]-[3], [8], [26]) certain approximations have been made. Some flow-induced terms can be shown to be small and have been neglected. Likewise, while it can be argued that the factor $3 / 2$ appearing in the second term of the right-hand side should be $5 / 2$ [27] (which would lead to relatively small changes in our final values for $V_{0}$ and $D$ ), the use of $3 / 2$ (instead of $5 / 2$ ) is still defensible in cases (such as ours) where the momentum balance of the plasma is influenced by processes such as turbulence [28]. Hence, in conformity with most of the current literature on empirical transport modeling, (2) and (3) have been used in our simulations.
}

The plasmas in IMS are usually very pure $\left(Z_{\text {eff }} \simeq 1\right)$, so a term modeling radiation losses is not included.

As can be seen, there is no equation governing the electron density. Instead, the "quasi-neutrality" approach is used. That is, the electron density is set equal to the ion density, $n_{e}=n_{i}=n$, and the electron particle flux is determined by the ion particle flux, $\Gamma_{e \perp}=\Gamma_{i \perp}=\Gamma_{\perp}$.

The way in which $S_{i}, Q_{e i}, P_{e, \mathrm{RF}}, P_{e, N}$, and $P_{i, N}$ are modeled will be discussed in Section III. Initially, the perpendicular particle and heat fluxes $\Gamma_{\perp}, q_{i \perp}$, and $q_{e \perp}$ were related to the gradients in temperature and density $\partial T_{e} / \partial r$, $\partial T_{i} / \partial r$, and $\partial n_{i} / \partial r$ via a matrix, having on its main diagonal the diffusion coefficient $D$ and the thermal conductivities $\kappa_{e}$ and $\kappa_{i}$. No attempt was made to include offdiagonal elements. Hence,

$$
\begin{aligned}
& \Gamma_{\perp}(r, t)=-D \frac{\partial n(r, t)}{\partial r} \\
& q_{e \perp}(r, t)=-\kappa_{e} \frac{\partial T_{e}(r, t)}{\partial r} \\
& q_{i \perp}(r, t)=-\kappa_{i} \frac{\partial T_{i}(r, t)}{\partial r} .
\end{aligned}
$$

As will be discussed in Section III, experimental measurements indicated the need to include convective (as opposed to purely diffusive) particle flow. Hence, (4) was modified to

$$
\Gamma_{\perp}(r, t)=-D \frac{\partial n(r, t)}{\partial r}+n(r, t) V(r)
$$

with $n(r, t) V(r)$ being the convective term. $V(r)$ is the convective flow in the minor radial direction. The physical mechanism(s) responsible for this convective flow will be discussed briefly in Section III.

Equations (1)-(3) are replaced by their equivalent finite difference equations, using a modified (i.e., slightly more implicit) Crank-Nicholson scheme [9]. $T_{e}, T_{i}$, and $n$ are then solved on a rectangular $(r, t)$ grid. The code uses variable time steps (determined by a specified maximum relative change in the fastest changing variable) and, if desired, a nonuniform spatial mesh.

\section{Modeling of the Physics Contained in the CODE}

\section{A. General Remarks}

A description of how the original code is organized can be found in [3]. In this section, we point out some changes along with a few important features needed further on.

The original code made a distinction between the main plasma region and a "scrape-off layer" (SOL) close to the edge. This is a remnant of its reactor character; all our results have been obtained without an SOL and with a uniform radial grid in the plasma. A consequence of this approach is the neglect of parallel particle and energy losses in the SOL due to divertors or limiters. Therefore, the modified code does not make a distinction between 
different poloidal harmonic numbers $l$ (except in the rotational transform) and/or different numbers of field periods, since the main effect of these parameters was restricted to these SOL-loss calculations.

The original code estimated the shift of the magnetic surfaces, by using the Chodura-Schluter three-dimensional MHD equilibrium code results [10]. In our application, we will neglect this shift, due to the low $\beta$ of plasmas being modeled (where $\beta$ is the ratio of plasma pressure to magnetic field pressure). Because the structure of the surfaces is not supposed to change substantially, it is not a cause for concern in our one-dimensional calculations, since all possible plasma-wall interactions (and divertor action) have been neglected.

The rotational transform profile $t(\rho)$ is specified by the user. This is done by supplying the coefficients of a polynomial fitting the desired profile:

$$
t(\rho)=\sum_{j=0}^{4} R_{j} \rho^{j}
$$

where the $R_{j}$ 's are coefficients to be specified, and $\rho$ stands for the normalized radial position, $r / a$ (where $a$ is the separatrix radius). A choice of coefficients in (8) for the transform was based on a fit to experimental measurements. The transform is zero on the magnetic axis and rises to 0.6 at the edge. A parabolic profile formula for the transform $t(\rho)=\left(R_{0}+R_{2} \rho^{2}\right)$ was used. In this case, $R_{2}=0.6$. If a nonzero transform can exist on the axis, which is the case if there is an equilibrium shift, the constant $R_{0}$ is nonzero. In our case, we estimate that $R_{0}$ has a value between 0.01 and $0.1 .\left(R_{j}=0\right.$ for $j \geq 3$.)

The user is also required to specify a desired (combination of) transport model(s). The transport coefficient at a particular $r$ value is then the linear combination of all the contributions from the transport processes specified. In all cases, the edge temperatures and density were held fixed and set to a relatively low value, as compared to the temperature and density values typical for the center of the discharge.

\section{B. Treatment of Neutrals}

In a plasma with a low degree of ionization, the neutral particles play a significant role, since the atomic processes in which they are involved are among the major factors determining $T_{e}, T_{i}$, and $n$. The physics of these processes and the related neutral species parameters (density, temperature, etc.) therefore require careful consideration.

The main objective of the code is the modeling of various electron cyclotron resonance heating (ECRH) experiments. In these, we can distinguish a breakdown phase, during which the cold neutral molecules are excited and dissociated, forming a cool, low-density plasma, and a heating phase, during which the RF heating leads to a subsequent increase in the value of the plasma parameters (temperature and density). Note that there are also other methods available to initiate breakdown, for example, a gun plasma source.
In our simulations, we do not attempt to model this initial breakdown phase. Rather, we start with an existing plasma (created by ECRH or perhaps some other means), and let it evolve towards a steady state under the influence of the ECRH. This allows us to use atomic hydrogen reaction-rate coefficient data in our modeling of particle and energy sinks and sources, an approach we will now justify in more detail.

"Target" steady-state plasma densities are between 1 $\times 10^{11}$ and $4 \times 10^{11} \mathrm{~cm}^{-3}$. The initial neutral density $n_{0}$, determined by the amount of gas that is puffed in at the beginning of a shot, is typically an order of magnitude larger, implying a degree of ionization $\eta$ of about 10 percent. The gas puffed in consists of hydrogen molecules, at room temperature, with typical speeds of about $2 \times$ $10^{5} \mathrm{~cm} \cdot \mathrm{s}^{-1}$. During the initial breakdown phase, several competing molecular collision processes take place. The most relevant for our discussion are [11]

- electron impact ionization processes

$$
e^{-}+H_{2} \rightarrow e^{-}+e^{-}+H_{2}^{+}
$$

and

- electron impact dissociation processes

$$
\begin{aligned}
& e^{-}+H_{2} \rightarrow e^{-}+H+H \\
& e^{-}+H_{2} \rightarrow 2 e^{-}+H+H^{+} .
\end{aligned}
$$

Assuming an initial low-density background plasma with a temperature of a few electronvolts, we can calculate the mean free paths $\lambda$ of an $\mathrm{H}_{2}$ molecule against dissociation and against ionization (where we define the mean free path of a particle of species 1 moving through a medium 2 as $\lambda=v_{1, \text { thermal }} / n_{2}\langle\sigma v\rangle$, with $\langle\sigma v\rangle$ the Maxwellian rate coefficient for the interaction under consideration [12]). The results (about 4 and $24 \mathrm{~m}$, respectively) indicate the importance of dissociation, as compared to molecular ionization. This can be made more quantitative by calculating the fraction $f$ of hydrogen molecules being dissociated instead of being ionized; $f$ turns out to be about 87 percent. Moreover, the few $\mathrm{H}_{2}^{+}$ions that are formed have a relatively small mean free path (approximately $40 \mathrm{~cm}$ ), after which they are dissociated into neutral atoms and/or ions. Thus, during the breakdown phase (which is not modeled by the code), the dominant process is a dissociation of the molecular hydrogen into neutral atoms and ions. The fraction of molecular ions is small and can be neglected. These considerations justify our use of atomic hydrogen reaction-rate coefficient data in our calculations of particle and energy sinks and sources.

It is also assumed that the neutral density has a flat radial profile and shows no $r$ dependence throughout a shot. This zero-dimensional treatment can be easily justified by evaluating the mean free paths of the neutral atoms, both against collision processes such as electron-impact ionization and charge exchange, and against collisions with other neutrals. Again, the results are of the order of several meters, much larger than the dimensions of the vac- 



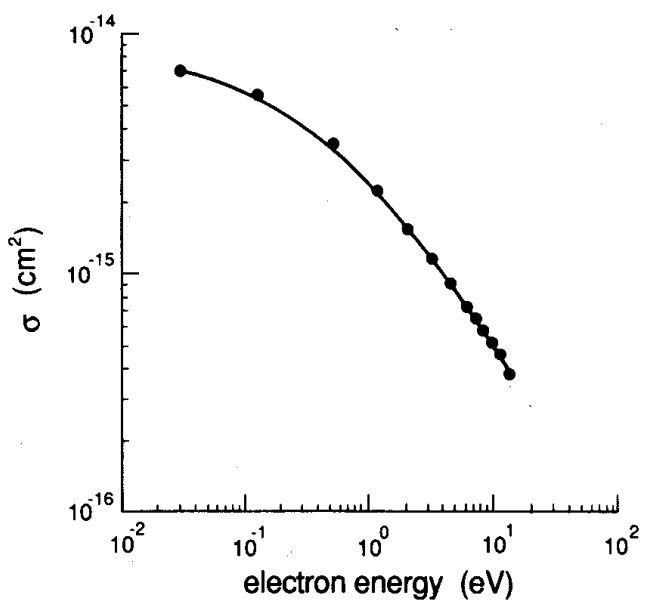

Fig. 1. Scattering cross section for electron-hydrogen atom elastic collisions.

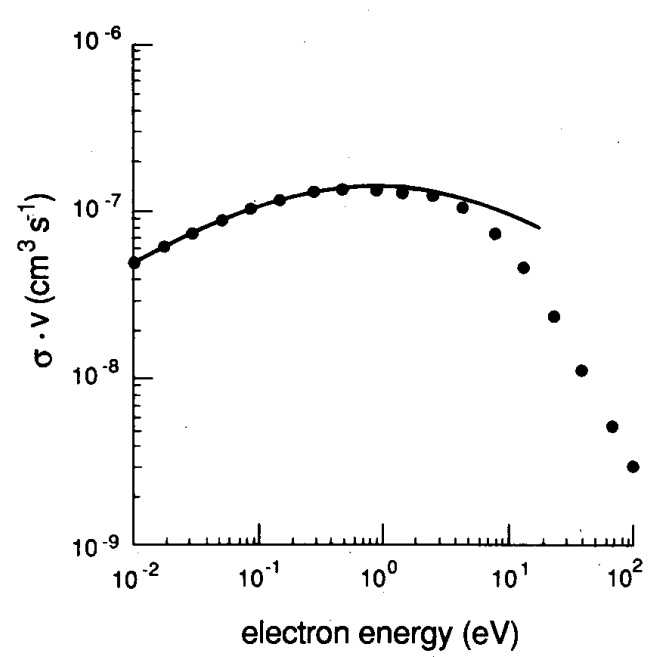

Fig. 2. Reaction-rate coefficient for electron-hydrogen atom elastic collisions.

where

$P_{e, N}^{\mathrm{eii}}(r)=n_{e}(r) n_{0}\langle\sigma v\rangle_{\mathrm{eii}} E_{l}$

$P_{e, N}^{\mathrm{ec}}(r)=n_{e}(r) n_{0}\langle\sigma v\rangle_{\mathrm{ec}} \frac{m_{e}}{m_{a}}\left(\frac{3}{2} T_{e}(r)-\frac{3}{2} T_{0}\right)$.

$E_{I}$ is the appropriate ionization energy $(13.6 \mathrm{eV}$ or higher). As can be seen, the lack of a term similar to the one in (11) indicates that the electrons originating from ionization are assumed to have zero energy.

Note also that the thermal equilibration between ions and electrons (the $Q_{e i}$ term of (2) and (3)) is modeled in the standard fashion (see, e.g., Trubnikov [18]).

\section{E. RF Heating}

The IMS plasmas that we have simulated are heated with ECRH. The ECRH power entering the vacuum chamber is not absorbed at a fixed radial location, but on a three-dimensional resonance absorption surface, the lo- cation of which depends on wave frequency, plasma density, etc. [19]. To model the RF power density imparted to the electron species $\left(P_{e, \mathrm{RF}}\right.$ in (2)) with a one-dimensional code, we have to estimate the amount of power absorbed as a function of radial position. $P_{e, \mathrm{RF}}$ is determined by the superposition of two effects: 1) possible effects of resonance and 2) the influence of the electron density.

The possible effects of selective absorption of RF power at resonance absorption surfaces are described by a dimensionless factor $W(r)$ (for example, a Gaussian or a bi-Gaussian), which contains the relative weight attributed to those flux surfaces (and hence $r$ values) that intercept the relevant absorption surface.

In the presence of plasma, we expect that more power will be absorbed in regions of higher electron density. We thus combine both effects into an expression for the net absorbed power as

$$
P_{e, \mathrm{RF}}(r)=C W(r) n_{e}(r)
$$

where $C$ is a normalization constant chosen in such a way that the total absorbed power integrated over the plasma volume is equal to the specified input power.

The ECRH experiments that are the subject of our simulations permit a further simplification of (15). Its justification requires a brief discussion of some aspects of a typical heating experiment. The waveguide launching RF power into IMS is located on top of, and perpendicular to, the machine. Thus, the heating wave propagates mainly perpendicular to the magnetic field. Heating experiments with both $X$ - and $O$-mode waves have been performed. Perhaps surprisingly, no significant differences in resulting plasma parameters were observed. This indicates that an analysis based on standard plasma wave theory (for example, mode conversion of the incoming $X$ mode wave at the upper hybrid resonance layer to electrostatic Bernstein waves which are reflected back towards the electron cyclotron resonance layer with subsequent absorption) might be inadequate.

It should also be noted that due to the low density of the plasmas involved, the ensuing single-pass absorption is small, and multiple reflections off the vacuum vessel walls are to be expected.

Hence, it can be argued that the vacuum vessel should be considered a multimoded cavity, in which the absorption of the injected RF fields is based on stochastic waveparticle interactions [11]. The main conclusion for our purposes is simply that no resonance layer effects have to be taken into account; hence, we can assume that $W(r)$ $=$ constant.

Finally, it must be realized that the code assumes 100 percent coupling, while in reality only a certain fraction of the power delivered by the ECH source is absorbed (the rest will be absorbed by the vacuum vessel). How large a fraction is hard to estimate, but in most of our runs, we specified a $1-\mathrm{kW}$ source and a coupling of no more than 10 percent, so the power effectively absorbed by the plasma was assumed to be less than $100 \mathrm{~W}$. 


\section{F. Transport Coefficients}

In the numerical results to be presented, we have investigated the extent to which different sets of transport models are adequate in simulating the hollow profiles mentioned before.

We have examined the effects of a neoclassical transport model on the evolution of the discharge. For typical IMS-like temperatures and densities, the neoclassical transport coefficients are in the Pfirsch-Schluter regime. Diffusion and thermal diffusivity coefficients in this regime are well known (see, e.g., [20]) and need not be repeated here.

Direct application of these transport coefficients to model IMS plasmas is questionable, for the following reasons. First of all, the physical reasoning leading to a Pfirsch-Schluter diffusion coefficient depends upon the assumption of a fully ionized plasma, which is not realized experimentally. Second, problems arise in the center of the discharge where the safety factor $q,\left(q=t^{-1}\right)$ becomes very large, and where the transport coefficients blow up accordingly, and at the edge, where the divertors present in IMS will have an influence on the transport. Third, the expressions for the diffusion and thermal diffusivity coefficients contain collision rates based upon charged particle-charged particle collisions, while in reality charged particle-neutral particle collisions are more numerous. Thus, although a neoclassical PfirschSchluter transport model has been used in some of our simulations, caution is needed when interpreting the results.

The use of a different transport model originated from the following experimental observations. Space-potential measurements in hydrogen discharges, both during and after the RF power injection, have shown that in the former phase, large variations in potential can occur, on a given magnetic surface [21]. The resulting electric field within the surface can drive an $\boldsymbol{E} \times \boldsymbol{B}$ convective flow that is directed radially outward.

Diffusion based on small-scale convective cell structures in the plasma (Okuda-Dawson transport) [22] has been included (although it cannot explain the hollow profiles). The effective Okuda-Dawson diffusion coefficient resulting from small-scale convection driven by potential gradients on a magnetic surface is given by

$$
D_{\mathrm{OD}}=\sum_{m} \frac{\left|E_{m}\right|^{2}}{B_{T}^{2}} \tau_{m}
$$

where

$B_{T}$ toroidal field strength,

$E_{m} \quad m$ th mode electric field strength, and

$\tau_{m}$ correlation time for the $m$ th mode

and summation is over the different modes obtained by Fourier-decomposing the electric field in poloidal angle. Fig. 3 shows a graph of the diffusion coefficient profile derived in this way for the hydrogen case.

A major drawback of the two transport models de-

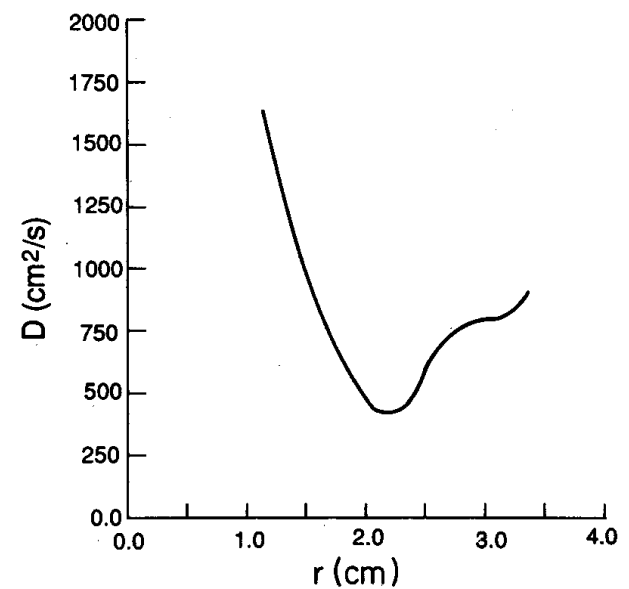

Fig. 3. Diffusion coefficient versus minor radius, based on Okuda-Dawson model.

scribed so far is that, by virtue of their purely diffusive character, it is impossible to create hollow profiles if the RF power density weighting factor $W(r)$ is constant in $r$. Even if the effect of resonance absorption surfaces as predicted by plasma wave theory is taken into account (by choosing $W(r)$ to be a bi-Gaussian with a maximum between the radial location of the upper hybrid and electron cyclotron resonance layer), the density profiles, although initially hollow, gradually fill in during the second half of the discharge, contrary to experimental observations.

For this reason, the particle flux $\Gamma_{\perp}$ was modified to include a convective term $n(r) V(r)$. In our choice of a $V(r)$ profile, we were guided by the following two points:

1) $V(r)$ must be zero at $r=0$ to avoid a physically unacceptable nonzero slope of the density profile at $r=0$. This follows directly from a simple analysis of the density balance equation in the region near $r$ $=0$.

2) Experimental measurements indicate a $V(r)$ profile that is zero at $r=0$, close to zero (or even slightly negative) at $r=a$, and peaks at intermediate values [23].

These observations led to a simple model for the radial profile of the convective velocity:

$$
V(r)=V_{0} \sin ^{2}[\pi r / a]
$$

An appropriate value for $V_{0}$ was determined heuristically. The experimental measurements mentioned before suggest a value for $V_{0}$ of about $500 \mathrm{~cm} / \mathrm{s}$.

\section{Comparison Between Experimental AND Numerical Results}

\section{A. Experimental Results}

The IMS device is an $l=3$, seven field-period modular stellarator, designed to match as closely as possible the parameters of the Proto-Cleo stellarator [24]. Table I summarizes the main parameters of IMS.

The code was used to model the hollow profiles ob- 
TABLE I

MaIN PARAMETERS OF IMS

\begin{tabular}{llll}
\hline \hline$R_{\text {major }}$ & $0.4 \mathrm{~m}$ & Magnetic Axis Radius & $0.4 \mathrm{~m}$ \\
$R_{\text {minor (coil) }}$ & $0.08 \mathrm{~m}$ & Plasma Aspect Ratio & 10.4 \\
Coil Aspect Ratio & 4.76 & $B_{0}$ & $3.0 \mathrm{kG}$ \\
Plasma Minor Radius & $0.04 \mathrm{~m}$ & Edge Transform & 0.6 \\
Flux Volume & $1.11 \times 10^{-2} \mathrm{~m}^{3}$ & Edge Ripple & $24.6 \%$ \\
Number of Field Periods 7 & &
\end{tabular}

served experimentally. A brief summary of the relevant experimental results obtained in IMS will be presented.

The capacitor bank power supply, feeding current to the magnets, has a "flat top" of $10 \mathrm{~ms}$. During this time, a $10-\mathrm{ms}$ pulse of up to $3 \mathrm{~kW}$ of $7.275-\mathrm{GHz} \mathrm{RF}$ power is applied to produce, heat, and maintain a hydrogen plasma.

Hollow density profiles are observed over a wide range of experimental conditions (e.g., for different magnetic field strengths). The profiles are fairly symmetrical with respect to $r=0$. The radial position of the outermost density peak is consistent with absorption between the electron cyclotron and upper hybrid resonance layer, although the similar results obtained with $X$-and $O$-mode RF fields cast doubt upon this mechanism being at the root of these peculiar profiles. The hollowness of the profile-defined as the maximum density divided by the density at the origin-takes on values in the range 1.5-10.

The electron temperature profile is somewhat hollow as well, but the uncertainties in the measurements preclude any definite judgment, and the fairly flat temperature profiles seen in our numerical simulations can be considered to be compatible with the experiments.

In our simulations, we shall concentrate upon the heating phase, and, in particular, upon the steady-state profiles obtained at the end of the $10-\mathrm{ms}$ period of ECRH. A summary of the plasma parameters to be matched by the code is as follows:

- electron temperatures between 5 and $10 \mathrm{eV}$

- ion temperatures between 2 and $4 \mathrm{eV}$

- densities between 1 and $4 \times 10^{11} \mathrm{~cm}^{-3}$

- hollowness between 1.5 and 10 .

\section{B. Numerical Results}

In this section, we will present the numerical results obtained in our efforts to simulate the hollow profiles discussed before. In all the runs to be discussed subsequently, the absorbed microwave power is set to a value in the range from 50 to $100 \mathrm{~W}$; higher values tend to produce too high electron densities, incompatible with the assumption of a weakly ionized plasma, and not in agreement with experimental findings. Neutral densities are usually set to a value of about $10^{12} \mathrm{~cm}^{-3}$. The energy lost by an electron upon ionization of a neutral has been set between $13.6 \mathrm{eV}$ and up to three times this value, so as to consider excitation of neutrals and other electron energy loss channels as well. Initial plasma densities were always set to be several orders of magnitude smaller than the neutral density.

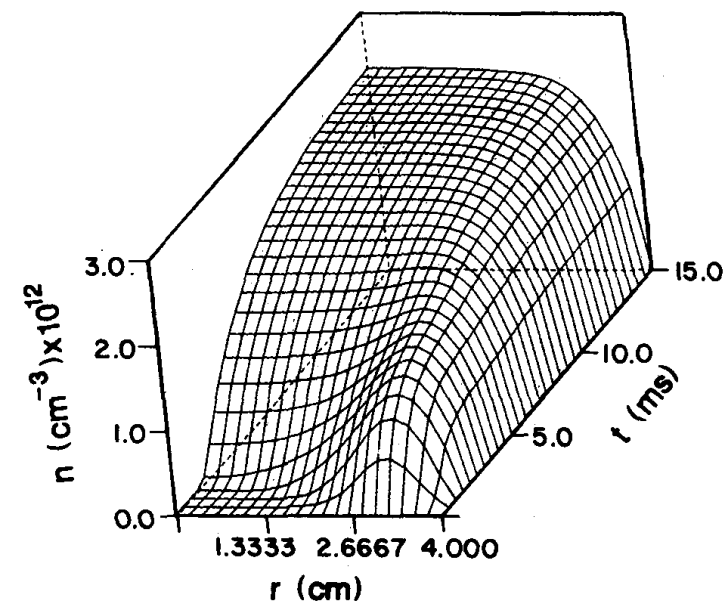

Fig. 4. Plasma density versus minor radius and time-case I-(a).

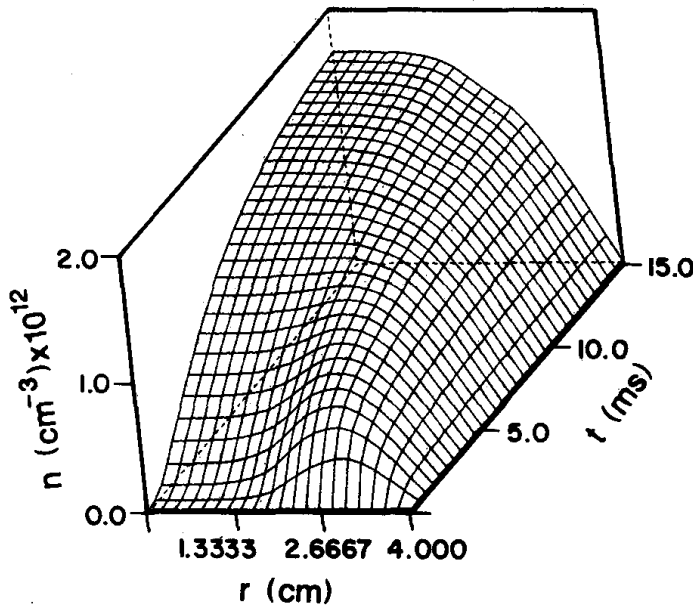

Fig. 5. Plasma density versus minor radius and time-case I-(b).

Before describing any specific results, we discuss, in general terms, the approach we have taken to simulate the experimental profiles. The code should be considered as a tool to judge the merits of different transport models thought to play a role in the formation of these profiles.

In this context, we have investigated, first, the combination of an RF-power deposition profile in which $W(r)$ is bi-Gaussian with either neoclassical Pfirsch-Schluter or Okuda-Dawson transport (but no convection), and second, whether the combination of an RF-power deposition profile in which $W(r)$ is constant with a transport model in which convection is included, allows hollow profiles.

We now proceed with a presentation of the simulation results for the following cases: case I-(a) Pfirsch-Schluter transport and (b) Okuda-Dawson transport; and case II-diffusive/convective transport combined.

Figs. 4 and 5 show the main results for cases I-(a) and I-(b), respectively: In both cases, the simulation lasted for $15 \mathrm{~ms}$ to allow the plasma to reach a steady state. The profiles are initially hollow but gradually fill in, in the course of the discharge. Note also that the final densities 


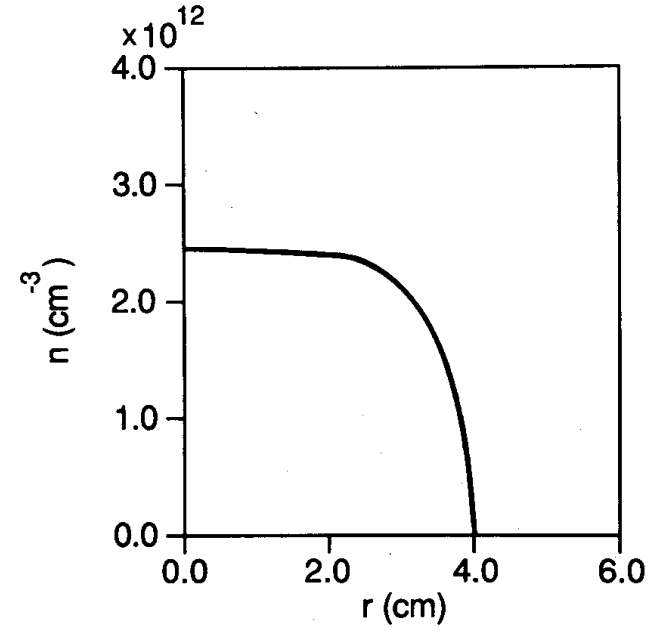

Fig. 6. Dependence of the steady-state density profile on minor radiuscase $\mathrm{I}-(\mathrm{a})$.

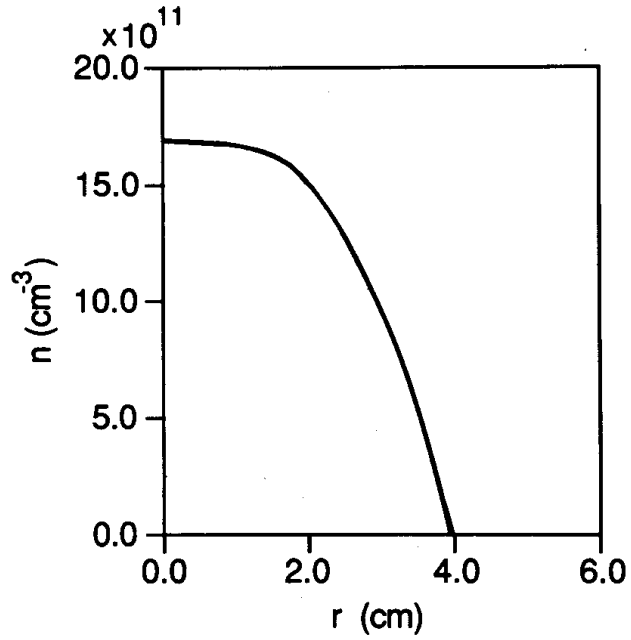

Fig. 7. Dependence of the steady-state density profile on minor radiuscase I-(b).

are at least a factor 3 too large compared with experimental values. For comparison with the results from case II, Figs. 6 and 7 show the final profiles, reached after $15 \mathrm{~ms}$. More interesting are the results for case II. Fig. 8 shows the time evolution of the density, while Fig. 9 depicts the final density profile (in this case, after $10 \mathrm{~ms}$ of RF heating). The final temperature profiles, both for electrons and ions, can be found in Fig. 10. Input parameters used to obtain Figs. 8-10 are the following:

- neutral density: $10^{12} \mathrm{~cm}^{-3}$

- ECRH power: $50 \mathrm{~W}$

- convective velocity as in (17), with $V_{0}=1000 \mathrm{~cm} / \mathrm{s}$

- diffusion coefficient $D=1750 \mathrm{~cm}^{2} / \mathrm{s}$.

The resulting plasma parameters can be summarized as follows:

- maximum density $4.53 \times 10^{11} \mathrm{~cm}^{-3}$

- minimum density (at $r=0$ ) $9.40 \times 10^{10} \mathrm{~cm}^{-3}$

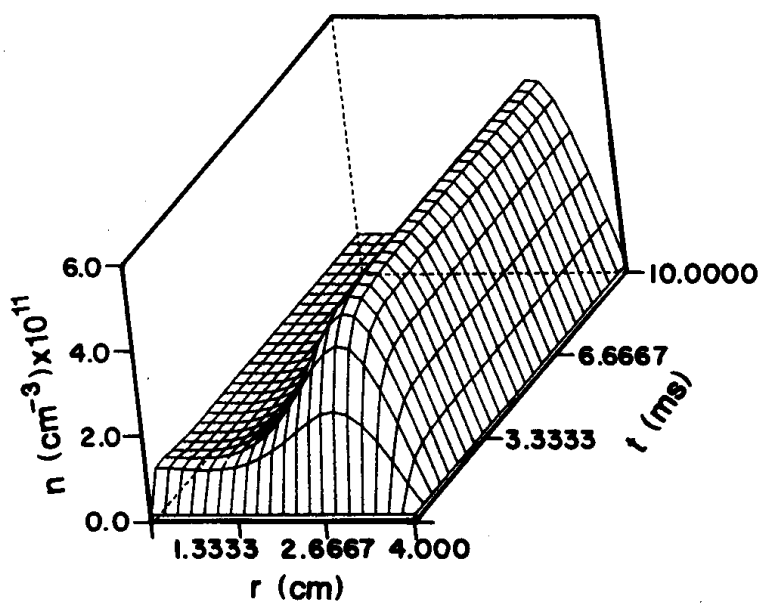

Fig. 8. Plasma density versus minor radius and time-case II.

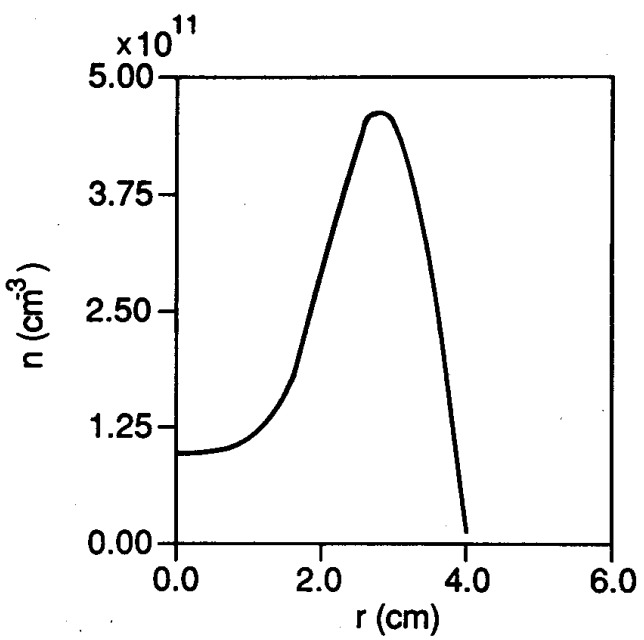

Fig. 9. Dependence of the steady-state density profile on minor radiuscase II.

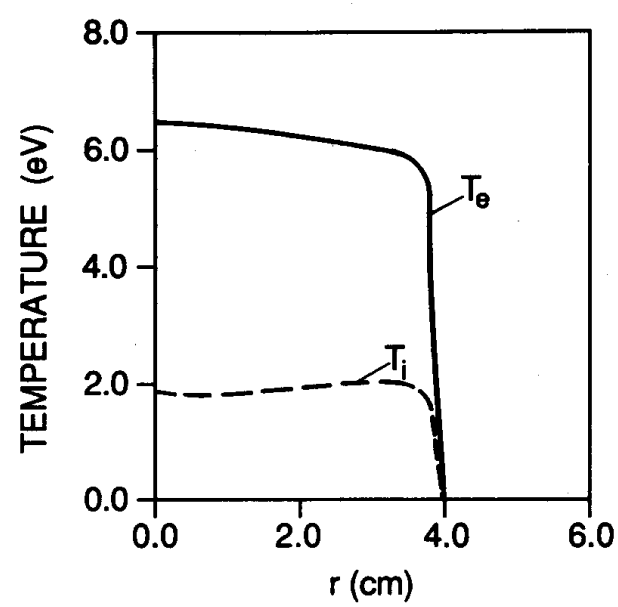

Fig. 10. Dependence of the steady-state temperature profiles on minor radius-case II. 
- corresponding hollowness 4.8

- average electron temperature $6 \mathrm{eV}$

- average ion temperature $2 \mathrm{eV}$.

All these values are in good agreement with the experimental observations. Both electron and ion temperature profiles are fairly flat, regardless of the precise value of the thermal diffusivities. The values of $V_{0}$ and $D$, however, have a strong influence on the final hollowness and the final average density.

\section{Conclusion}

In this paper, we have discussed the physics contained in the modified WHISTEL stellarator reactor transport code and the first results we have obtained with it.

The main application to date was prompted by the experimental observation of hollow density profiles in IMS, a small modular stellarator. We have thus initiated a numerical study of the interplay between diffusion, convection, atomic processes, and ECRH, and how these factors determine the possible formation of steady-state hollow density profiles.

To model the relevant IMS experiments efficiently, the neutral species can be modeled with a fixed density radially and temporally. The neutral temperature is treated zero dimensionally as well, but is allowed to evolve in time. A discussion of typical mean free paths in IMS shows that in this device the neutral-particle model described is well justified.

Since it was not our intention to model the initial breakdown phase, during which most of the dissociation of molecular hydrogen into neutral atoms and ions takes place, the use of atomic reaction rate coefficients could be justified.

Two diffusive transport models have been investigated, one of them neoclassical in nature, the other one based on the small-scale convective cell structures in the plasma that can be associated with an $\boldsymbol{E} \times \boldsymbol{B}$ particle drift that is directed radially outward. A purely convective term was then included in the particle balance equation. A combination of a constant diffusion coefficient with a convective velocity that exhibits a $\sin ^{2}(r)$ dependence (as suggested by experimental measurements) gives rise to hollow density profiles in which all the plasma parameters for which experimental values are available are in good agreement with the observed values. No discussion of the origin of the convective flow was given. This will be treated in a forthcoming paper [25].

\section{ACKNOWLEDGMENT}

The authors wish to thank A. W. Bailey for providing them with the original WHISTEL code.

\section{REFERENCES}

[1] J. T. Hogan, "Multifluid tokamak transport models," in Methods in Computational Physics, vol. 16, J. Killeen, B. Alder, S. Fernbach, and M. Rotenberg, Eds. New York: Academic, 1976, p. 131.

[2] C. E. Singer et al., "BALDUR: A one-dimensional plasma transport code," Princeton Univ. Rep., Princeton, NJ, PPPL 2073, July 1986.

[3] A. W. Bailey, G. A. Emmert, and K. J. Lee, "WHISTEL, a stellarator transport code," Univ. Wisconsin, Madison, Fusion Design Memo-477, Oct. 1982.

[4] D. T. Anderson, J. A. Derr, and J. L. Shohet, "The interchangeable module stellarator," IEEE Trans. Plasma Sci., vol. PS-9, no. 4, p. 212, Dec. 1981

[5] C. A. Storlie et al., "Determination of particle convective and diffusive transport coefficients in IMS," in IEEE COPS Conf. Rec. Abstracts, June 1987, p. 32.

[6] S. I. Braginskii, "Transport processes in a plasma," in Reviews of Plasma Physics, vol. 1. New York: Consultants Bureau, 1965, p. 205.

[7] F. L. Hinton and R. D. Hazeltine, "Theory of plasma transport in toroidal confinement systems," Rev. Mod. Phys., vol. 48, no. 2, part 1, p. 239, Apr. 1976.

[8] W. A. Houlberg, S. E. Attenberger, and L. L. Lao, "Computational methods in tokamak transport," Oak Ridge Nat. Lab., Oak Ridge, TN, Rep. TM-8193, June 1982.

[9] L. Lapidus and G. F. Pinder, Numerical Solution of Partial Differential Equations in Science and Engineering. New York: Wiley, 1982.

[10] R. Chodura and A. Schluter, Inst. für Plasma Phys., Rep. 1/180, 1980.

[11] L. C. S. Goes, Ph.D. dissertation, Univ. Wisconsin, Madison, 1986.

[12] T. J. Dolan, Sr., Fusion Research, Vol. 1: Principles. New York: Pergamon, 1982.

[13] R. L. Freeman and E. M. Jones, "Atomic collision processes in plasma physics experiments," Culham Lab, Abingdon, Oxfordshire, U.K., Rep. CLM-R137, May 1974.

[14] R. T. Brackmann, W. L. Fite, and R. H. Neynaber, "Collisions of electrons with hydrogen atoms. III. Elastic scattering," Phys. Rev., vol. 112 , no. 4 , p. 1157 , Nov. 1958.

[15] R. H. Neynaber, L. L. Marino, E. W. Rothe, and S. M. Trujillo, "Scattering of low-energy electrons by atomic hydrogen," Phys. Rev., vol. 124, no. 1, p. 135, Oct. 1961.

[16] R. P. McEachran and P. A. Fraser, "d-wave contribution to electron-hydrogen atom scattering," Can. J. Phys., vol. 38, p. 317, 1960.

[17] Yu. S. Gordeev, A. N. Zinov'ev, and M. P. Petrov, "Recombination of hydrogen in a quasi-stationary thermonuclear plasma," JETP Lett., vol. 25, no. 4, p. 204, Feb. 1977.

[18] B. A. Trubnikov, "Particle interactions in a fully ionized plasma," in Reviews of Plasma Physics, vol. 1. New York: Consultants Bureau, 1965, p. 105.

[19] W. D. D'Haeseleer, J. L. Shohet, and K. R. Audenaerde, "Threedimensional ray tracing in toroidal stellarators," IEEE Trans. Plasma Sci., vol. PS-15, no. 1, p. 28, Feb. 1987.

[20] V. E. Golant, A. P. Zhilinsky, and I. E. Sakharov, Fundamentals of Plasma Physics. New York: Wiley, 1980.

[21] D. T. Anderson et al., "Potential structure in IMS," Bull. Amer. Phys. Soc., vol. 30, no. 9, p. 1577, Oct. 1985.

[22] H. Okuda and J. M. Dawson, "Effect of magnetic shear on convective plasma transport," Phys. Fluids, vol. 16, no. 9, p. 1456, Sept. 1973.

[23] P. K. Trost, private communication, June 1987.

[24] R. A. E. Bolton et al., "Plasma containment in medium and high shear stellarators," Phys. Fluids, vol. 14, no. 7, p. 1566, July 1971.

[25] J. N. Talmadge et al., to be published.

[26] W. A. Houlberg and R. W. Conn, "Fluid simulation of large tokamak plasmas using a variable spatial mesh, variable time step implicit procedure," Nucl. Sci. Eng., vol. 64, p. 141, Sept. 1977.

[27] D. W. Ross et al., "Thermal and particle transport in tokamaksTheoretical models for ignition studies," Univ. Texas Fusion Res. Center, Austin, Rep. 295, June 1987.

[28] W. A. Houlberg, private communication, Dec: 1987. 